\title{
O PROBLEMA DA INDUÇÃO SOB A ÓTICA DO BIG DATA: UM ENSAIO A PARTIR DOS ARGUMENTOS DO FILÓSOFO KARL POPPER
}

\author{
Marcos Luiz Lins Filho ${ }^{1}$ \\ Universidade Federal do Rio Grande do Norte - UFRN \\ mluizlins@ufrn.edu.br \\ Manoel Veras de Sousa Neto ${ }^{2}$ \\ Universidade Federal do Rio Grande do Norte - UFRN \\ manoel.veras@uol.com.br
}

\begin{abstract}
Resumo
A busca pelo conhecimento permanecerá sempre viva entre os seres humanos mantendo ativos os debates e as discussões. Nesse contexto, o uso de Big Data nas pesquisas científicas reacende a discussão sobre o uso da indução como base para a construção do conhecimento científico. Esse ensaio apresenta uma análise teórica acerca do problema da indução sob a ótica do Big Data. Para tanto, tomou-se como base para o contraponto os argumentos apresentados pelo filósofo Karl Popper. Conclui-se que, sob a ótica do uso de Big Data, o método indutivo não apresenta inconsistências e os enunciados universais podem ser inferidos a partir de enunciados singulares, contrariando a ideia de Popper e reforçando a teoria indutivista.
\end{abstract}

Palavras-chave: E-Science. Big Data. Problema da Indução. Karl Popper.

\section{THE PROBLEM OF INDUCTION UNDER THE BIG OPTICAL DATA: AN ESSAY FROM THE PHILOSOPHER KARL POPPER'S ARGUMENTS}

\begin{abstract}
The search for knowledge will always live among humans keeping active debates and discussions. In this context, the use of Big Data in scientific research rekindles the discussion about the use of induction as the basis for the construction of scientific knowledge. This paper presents a theoretical analysis of the problem of induction from Big Data. To this end, the arguments put forward by the philosopher Karl Popper were taken as the basis for the counterpoint. It is concluded that, from the perspective of the use of Big Data, the inductive method presents no inconsistencies and the universal statements can be inferred from singular statements, contrary to the idea of Popper and reinforcing the inductive theory.
\end{abstract}

Keywords: E-Science. Big Data. Induction Problem. Karl Popper.

${ }^{1}$ Graduação em Ciência da Computação pela Universidade Federal de Campina Grande (2003). Especialização em Segurança de Redes e Criptografia pela Universidade Federal Fluminense (2009). Mestrado em Ciência da Computação pela Universidade Federal de Pernambuco (2011). Especialização em Gestão de Tecnologia da Informação na Universidade Federal do Rio Grande do Norte (2013). Atualmente é Doutorando em Administração pela Universidade Federal do Rio Grande do Norte.

${ }^{2}$ Doutorado em Administração pela Universidade de São Paulo - USP (2001), mestrado em Engenharia Elétrica pela Universidade Estadual de Campinas - UNICAMP (1985) e graduação em Engenharia Elétrica pela Universidade Federal do Rio Grande do Norte - UFRN (1983).

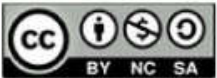

Esta obra está licenciada sob uma Licença Creative Commons Atribuição 4.0 Internacional (CC BY-NC-AS 4.0). LOGEION: Filosofia da informação, Rio de Janeiro, v. 5 n. 1, p. 6-19, set.2018/fev. 2019 


\section{INTRODUÇÃO}

A busca do conhecimento sempre esteve presente entre as principais aspirações dos seres humanos. A história retrata que essa busca inicia a partir do estudo da cosmologia, em que o objetivo era o entendimento da origem e dos fenômenos do universo. O primeiro momento, marcado pelas reflexões focadas na religião e na mitologia.

Num segundo momento, um dos marcos para evolução do conhecimento humano foi o surgimento da Teoria, que buscava a compreensão racional, a partir da organização, integração e dinamização do conhecimento (VIANA; PEREIRA, 2007).

Partindo para Antiguidade Clássica, considerada como uma nova fase na construção do conhecimento, o destaque é o filósofo Aristóteles (384 - 332 a.C.), que estabelece um método de pesquisa através de um processo sistematizado para a produção de conhecimento. $\mathrm{O}$ chamado método indutivo-dedutivo.

Nessa época, o filósofo ao estabelecer os tratados referentes ao raciocínio formal, faz menção a indução como parte da busca pelo conhecimento universal a partir da observação de casos particulares. Na obra Primeiros analíticos, a indução surge basicamente em forma de silogismo. Já na obra Segundos analíticos, Aristóteles deixa claro a busca pela verdade universal e sem restrições. Nesse contexto, a indução que busca a universalidade está associada a intuição. Para Aristóteles, a intuição é sempre verdadeira e ela que apreende as premissas primárias (GRÁCIO, 2005).

Durante muitos anos, a concepção de produção de conhecimento esteve fundamentada na teoria indutivista de Aristóteles. Porém, a visão indutivista só se torna hegemônica para a ciência a partir da sistematização feita por Bacon (1984 apud VIANA, 2007, p. 5), quando afirma que "toda ciência começa pela observação, para depois proceder vagarosa e cautelosamente à formulação de teorias" (POLYA, 1978). Para Bacon (1984 apud VIANA, 2007, p. 5), a indução exige o cumprimento de uma sequência de etapas que vai desde a observação até a confirmação ou não de uma hipótese provisória.

Num contexto mais amplo das ciências empíricas, o conceito de indução pode ser dado a partir da definição do filósofo Popper (1972), que a descreve como uma inferência que conduz a partir de enunciados singulares para chegar a enunciados universais (POPPER, 1972). Há que se pontuar, que a hegemonia estabelecida pela teoria indutivista em relação a dedutivista, durante este período, muito se deve ao fato da indução, ao contrário da dedução, estabelecer uma demarcação clara entre o que é ciência e metafísica (OLIVA, 1990). Esta demarcação 
passou a denotar aos indutivistas certa superioridade do ponto de vista explicativo e esclarecedor da ciência (SILVEIRA; OSTERMANN, 2002).

Segundo Silveira e Ostermann (2002), a partir de 1930 passou-se a questionar fortemente a concepção fundamentada na teoria indutivista. A incapacidade da indução em justificar os ditos enunciados universais a partir dos enunciados singulares, fez surgir o questionamento quanto a validade das inferências indutivas. Segundo Hume (2002), independente das experiências tidas, não é totalmente válido realizar qualquer inferência de uma possível experiência futura com base nas experiências passadas. A partir daí, inicia-se mais intensamente os debates e questionamentos acerca da validade da indução pelos filósofos e epistemólogos contemporâneos.

Por um lado, há críticas contemporâneas como as sustentadas por Popper (1975); Kuhn (1978); Hanson (1979) e Feyeraband (1993) que se fundamentam na inseparabilidade entre os pressupostos teóricos e as observações e que sustentam uma oposição ao método indutivo para desenvolvimento científico (SILVEIRA; OSTERMANN, 2002). Na outra ponta, vê-se um esforço de indutivistas em buscar soluções alternativas para o problema da indução como Mill (1979); Carnap (1962); Hume (2002) e Russel (1939) (VIANA; PEREIRA, 2007).

Nesse contexto, a emergência da chamada e-Science, nas últimas décadas do século XX, fez com que a ciência passasse a se valer cada vez mais da vertente computacional na exploração e uso de grandes volumes de dados para auxiliar na construção de novas teorias, experimentos e simulações (ALBAGLI; APPEL; MACIEL, 2013 apud GRAY, 2007; BELL; HEY; SZALAY, 2009). Do ponto de vista de produção do conhecimento, o conceito de Big Data trouxe consigo uma nova perspectiva para a ciência, em especial para os indutivistas, pois retoma a ideia básica do raciocínio indutivo quando se propõe a construir conhecimento e fazer predições a partir de enunciados singulares.

O termo Big Data possui diversas definições, porém não existe um conceito preciso na literatura. Vários autores o tratam como um termo que se refere a grandes quantidades de dados (KATAL; WAZID; GOUDAR, 2013; KAISLER et. al., 2013; SAGIROGLU; SINANC, 2013). Porém, além do volume massivo de dados, apresenta também como características o crescimento contínuo e em alta velocidade e uma grande variedade dos dados. Essas características particulares do Big Data, remetem a necessidade de desenvolver novas formas de lidar com os grandes volumes de dados na pesquisa científica, dando novo rumo a e-Science.

O Big Data traz uma nova visão e diversos desafios para a pesquisa científica, pois se propõe a analisar volumes massivos de dados que se modificam com grande velocidade e possuem grande variedade. Além disso, retoma a discussão quanto a validade da indução dentro 
de uma nova perspectiva de construção do conhecimento científico, na qual o Big Data é o elemento central.

Partindo dessa nova realidade, este ensaio fará uma discussão teórica do papel do Big Data dentro de uma nova perspectiva de construção do conhecimento científico, sob o olhar da teoria indutivista, dos seus problemas e soluções. Inicialmente, para situar o leitor, será apresentada uma contextualização a partir do problema da indução. Em seguida, discute-se o papel do Big Data no contexto da pesquisa científica, fazendo uma breve leitura dos desafios que o Big Data traz para a e-Science. Na sequência, apresenta-se o problema da indução, sob a ótica do Big Data e fazendo um contraponto aos argumentos apresentados pelo filósofo Popper (1972). Finalmente, na última seção são apresentadas as considerações finais.

\section{O PROBLEMA DA INDUÇÃO}

Segundo Popper (1972), uma inferência é dita indutiva quando parte de enunciados particulares, tais como as descrições dos resultados das observações ou experimentos, para enunciados universais, tais como hipóteses ou teorias.

Porém, o chamado problema de indução está no fato de que, para a indução, as premissas verdadeiras não garantem a verdade da conclusão. Vários filósofos e epistemólogos já negaram o fato de que o conhecimento científico esteja unicamente baseado em observações (SILVEIRA; OSTERMANN, 2002).

Um exemplo contrário pode ser o do filósofo Hume (2006), que afirma que a experiência passada provê informação imediata e segura somente acerca dos objetos que lhes foram dados, e apenas durante um certo período de tempo, mas a extensão dessa experiência a tempos futuros e a outros objetos é passível de erros.

O filósofo ainda reforça seu posicionamento contrário a validade da indução quando afirma que todas as nossas conclusões experimentais decorrem da suposição de que o futuro estará em conformidade com o passado. Portanto, tentar provar a última suposição, por argumentos prováveis, por argumentos referentes à existência, consiste, certamente, em girar num círculo vicioso e dar por admitido o que precisamente se problematiza (HUME, 2006).

Já Popper (1972), deixa claro seu posicionamento quanto ao problema da indução quando afirma está longe de ser óbvio de um ponto de vista lógico, haver justificativa no inferir enunciados universais de enunciados singulares independentemente de quão numerosos sejam estes; com efeito, qualquer conclusão colhida desse modo sempre pode revelar-se falsa. 
Filósofos e epistemólogos admitem o problema da indução, porém os debates e estudos acerca do tema não se esgotam. Outros estudiosos oferecem respostas para o problema da indução, mesmo que estas aparentemente ainda não contemplem, até o presente momento, a solução definitiva para o problema. No entanto, apresentam até certo ponto, uma aceitação por parte de alguns defensores da teoria indutivista dentro da comunidade científica. Entre as propostas, destacam-se dois grupos: os que defendem a rejeição da indução como forma de construção do conhecimento e apresentam novas teorias para resolução do problema da indução e os que trabalham numa proposta de reconstrução da teoria indutivista apresentando alternativas para minimizar o problema da indução.

Dentre os mais influentes entre os contemporâneos, o filósofo Popper (1972), tem se destacado por fazer parte do primeiro grupo que defende a rejeição da indução como forma de construção do conhecimento. Em seu discurso de rejeição da indução por repetição, afirma que "inferências que levam a teorias, partindo-se de enunciados singulares verificados por experiência são logicamente inadmissíveis" (POPPER, 1972).

Para Popper (1981), a indução só se tornaria um procedimento válido se em vez de buscar um enunciado verdadeiro ou lei natural e universal, houvesse uma substituição por um objetivo mais modesto, qual seja o de se chegar a um enunciado da probabilidade.

A partir dessas novas ideias, Popper (1972), desenvolve a teoria falseacionista e o método denominado hipotético-dedutivo. A teoria falseacionista contraria as demais teorias científicas até então vigentes, pois não busca provar que hipóteses, teorias e conjecturas são verdadeiras e sim demonstrar que são falseáveis. De acordo com o autor, a teoria que não for refutada por qualquer acontecimento concebível não é tida como científica, sendo, portanto, a falseabilidade, o seu critério para definição do status científico (POPPER, 1972).

A teoria falseacionista proposta por Popper (1972), deixa clara sua posição contrária em relação a existência do conhecimento científico pleno e verdadeiro, atribuindo aos testes, o poder de determinar a validade de uma teoria. Porém, não busca com isso eliminar o método indutivo do processo de construção do conhecimento, e sim torná-lo mais seguro e robusto, dando respostas ao problema da indução.

No grupo dos que buscam a reconstrução da teoria indutivista através de alternativas para o problema da indução, destacam-se John Stuart Mill, Hans Reichenbach, Rudolph Carnap e Bertrand Russel.

Para Mill (1979), a base teórica do problema de indução está focada na causalidade que ele considera como sendo universal e co-extensiva a todos os fenômenos sucessivos, isto é, a regularidade resulta de regularidades parciais. Dessa forma, segundo o autor é possível fazer 
inferências a partir de observações particulares que obedecem a uma uniformidade, que as observações futuras em todos os casos obedecerão a uniformidade observada nos primeiros (VIANA; PEREIRA, 2007).

Já Reichenbach (1938 apud VIANA; PEREIRA, 2007), propõe uma solução diferenciada partindo da inversão do conceito de indução clássico. A proposta é que se busque confirmar através das observações particulares uma generalização anteriormente verificada. Esse critério de verificabilidade corrobora com a teoria falseacionista de Popper (1972).

Já Carnap (1950 apud GRÁCIO, 2005), se baseia em um processo lógico para justificar a indução. A lógica é baseada no uso de probabilidades e, consequentemente, num conceito de grau de confirmação. O grau de confirmação avalia de forma probabilística o quanto uma hipótese está suportada por uma evidência.

Russell (1939 apud VIANNA; PEREIRA, 2007), faz referência a indução através de uma distinção entre inferência do tipo dedutiva e indutiva. Segundo Russell (1939 apud VIANNA; PEREIRA, 2007), para o conhecimento evoluir é necessário fazer uso de inferências do tipo indutiva, porém a confirmação da validade desse conhecimento e confirmação das teorias só é possível se utilizarmos inferências do tipo dedutiva. A indução é aceita por Russel (1939 apud VIANNA; PEREIRA, 2007), como algo que não se pode demonstrar de forma logicamente válida, porém também não pode ser provado inválido.

Outras duas propostas estão fundamentadas numa defesa pragmática da teoria indutiva e numa justificação como um pseudoproblema. A primeira se baseia no fato de que na ausência de argumentos que possibilitem chegar a conclusões verdadeiras, deve-se justificar as decisões a partir do efeito prático do conhecimento formulado através dos argumentos existentes. Esse tipo de proposta denominada de defesa pragmática é defendida por Charles S. Peirce e também por Hans Reichenbach (GRÁCIO, 2005).

A segunda proposta, defendida por Edwards (1949), afirma que a busca por uma justificativa racional para o problema de indução é uma tarefa impossível de se cumprir (EDWARDS, 1949). A impossibilidade parte do pressuposto de que, sendo a indução uma operação não dedutiva, torna-se inapropriado exigir que ela satisfaça as condições esperadas para uma dedução lógica. $\mathrm{O}$ argumento tem como base o uso inapropriado de critérios de avaliação entre domínios diferentes, portanto, os critérios tornam-se inadequados (GRÁCIO, 2005). 


\section{O BIG DATA E OS NOVOS RUMOS DA E-SCIENCE}

Assim como o tempo, a ciência não pode deixar de evoluir. Conforme já dizia Kuhn (1962), a forma como se faz ciência deve evoluir e passar por significativas mudanças, aprimoramentos e refinamentos na sua metodologia de trabalho, englobando a inserção de novas ferramentas e instrumentos de observação do mundo e novos paradigmas de estruturação do pensamento científico (KUHN, 1962).

Nesse contexto, nos últimos anos temos experimentado uma mudança radical na forma como se passou a fazer ciência através do uso massivo da componente computacional para auxiliar e promover maior abrangência do ponto de vista de complexidade, flexibilidade e agilidade nas pesquisas científicas.

O termo e-Science vem designar "a ciência crescentemente desenvolvida por meio de colaborações globalmente distribuídas e viabilizadas pela Internet, usando grandes coleções de dados, recursos computacionais em larga escala e visualização de alto desempenho" (ALBAGLI; APPEL; MACIEL, 2013 apud TAYLOR, 2002).

O termo e-Science mantem relação com o termo Big Data pelo fato de compartilharem a característica de trabalhar com grandes volumes de dados. Mas, o Big Data pode ser visto como um novo componente da e-Science, pois agrega a velocidade e variedade como características, isso o diferencia dos grandes volumes de dados estáticos. São os chamados $3 \mathrm{Vs}$ do Big Data: volume, velocidade e variedade (SINGH, 2011).

Segundo a Nature (2008), Big Data representa o progresso dos processos cognitivos humanos. Normalmente, são conjuntos de dados em que o tamanho vai além da capacidade das atuais tecnologias, métodos e teorias para captura, manipulação e processamento num tempo razoável.

As tecnologias tradicionais de processamento de dados, como os bancos de dados e datawarehouse estão inadequadas para lidar com a quantidade de dados que estamos demandando (CHEN et. al., 2013). Além disso, nenhuma dessas plataformas possui ferramental necessário para visualização e manipulação de dados que contemplem esse novo contexto (MADDEN, 2012).

Diante dessa nova realidade, o Big Data traz para a e-Science diversos desafios que devem direcionar os novos rumos da pesquisa científica utilizando a vertente computacional. Há que se responder a diversas perguntas como: Como lidar com a variedade de dados do Big Data na pesquisa científica? Que ferramentas utilizar para coleta, tratamento, análise e visualização? Como lidar com questão da velocidade de mudança e de crescimento dos dados? 
Como definir um ponto de parada na coleta de dados para então iniciar os demais processos subsequentes da pesquisa científica? Que métodos utilizar para analisar os dados? Como lidar com a questão do teste de validade de uma teoria, se antes de comprová-la ou testá-la, a mesma já pode estar invalidada pela presença de novos dados?

Não é pretensão deste ensaio esgotar, nem mesmo elencar todos os desafios e perguntas que a adoção do Big Data impõe a construção do conhecimento dentro do contexto da e-Science e da construção do conhecimento científico. A discussão aqui apresentada, busca mostrar o Big Data como um direcionador de mudanças no futuro da pesquisa científica, em especial, ao fato de retomar as discussões acerca da teoria indutivista, visto que essa é a teoria que fundamenta a construção de conhecimento a partir do Big Data.

É sabido que assim como os desafios impostos pelo Big Data à e-Science, os debates em torno da validade da indução permanecem ativos e parecem não esgotar. Nesse contexto, um dos trabalhos de destaque entre os filósofos contemporâneos e críticos do indutivismo é o trabalho de Popper. Popper (1972), apresenta a chamada teoria falseacionista, e descreve o método hipotético-dedutivo, que propõe uma solução racional para o chamado problema da indução.

A próxima seção apresenta uma discussão acerca do problema da indução, fazendo uma análise sob a ótica do uso do Big Data na construção do conhecimento científico a partir de um contraponto a alguns argumentos apresentados pelo filósofo Karl Popper (0000), acerca deste problema.

\section{O PROBLEMA DA INDUÇÃO E O BIG DATA}

Dentre as justificativas apresentadas pelos diversos filósofos e epistemólogos para o problema da indução, a solução apresentada por Popper (1972), até hoje se mantém em destaque no meio científico por ser uma abordagem que faz uso de um aparato metodológico para propor a solução do problema da indução. Ao propor sua solução, o autor enfatiza que existem dois pontos fundamentais que remetem ao que ele denomina por: problema lógico e problema psicológico.

O método proposto parte de uma solução totalmente dedutiva, deixando clara a visão de Popper (1972), sobre a impossibilidade de explicar a indução de forma pragmática (LISTON, 2001). Toda a argumentação proposta pelo filósofo exclui o problema psicológico, que posteriormente é retomado por Hume (2006 ou 2002) quando o mesmo apresenta suas ideias sobre hábito e crença. 
Tal postura, assumida por Popper (1972), o diferencia com relação aos demais filósofos que se propuseram justificar o problema da indução. A grande maioria dos filósofos sempre buscou a justificativa através do método indutivo. Tal método, não resolve o cerne da questão levantada pelo problema da indução do ponto de vista da racionalidade científica.

A justificativa de Popper (1972), ao contrário, busca mostrar através do que ele chama de teoria falseacionista e do método hipotético-dedutivo, que o problema da indução pode ser resolvido de forma metodológica e que o progresso do conhecimento na ciência se dá de forma racional e crítica (LISTON, 2001).

Para discussão do problema de indução sob a ótica do Big Data, os argumentos propostos pelo filósofo Popper (1972), para a solução do problema de indução serão apresentados e em seguida avaliados no contexto do uso de Big Data.

O primeiro argumento de Popper (1972), apresenta sua crítica a teoria indutivista que defende a validade e verdade dos enunciados universais derivados a partir de enunciados singulares:

“Ora, está longe de ser óbvio, de um ponto de vista lógico, que nós estamos justificados ao inferir enunciados universais a partir de enunciados singulares, independentemente de quão numerosos sejam estes; qualquer conclusão extraída desse modo sempre pode revelar-se falsa: não importa quantos casos de cisnes brancos nós possamos observar, isso não justifica a conclusão de que todos os cisnes são brancos" (POPPER, 1972).

No contexto do Big Data, a argumentação de Popper (1972) é inválida se levarmos em conta a afirmação de que o conhecimento não é gerado a partir dos enunciados particulares. A origem da construção do conhecimento a partir do Big Data vem da indução clássica, onde o acúmulo massivo de dados (enunciados singulares), forma a base para as inferências que possibilitarão a construção do conhecimento (enunciados universais).

Sob o olhar do Big Data, a extração de conclusões a partir dos enunciados singulares possui validade e pode ser justificada. $\mathrm{O}$ critério de validade se baseia no fato de que ao conjunto de enunciados singulares é atribuído o conceito estatístico de População e não de uma Amostragem.

Tal fato, resulta na eliminação do problema de indução apontado inicialmente por David Hume e que até hoje é discutido pelos filósofos. Considerando que todos os enunciados singulares possíveis estão reunidos formando o Big Data, pode-se inferir a partir dos enunciados singulares um enunciado universal, sendo que este, não é passível de contestação a partir do problema da indução, visto que a inferência indutiva pode, por sua vez, ser verificada a partir de um método dedutivo, mantendo assim a racionalidade do conhecimento científico. 
Sobre o critério de falseabilidade, Popper (1972), afirma que nenhuma teoria pode ser verificada a partir da experiência (enunciados singulares), porém através de testes é possível refutar uma teoria. Popper (1972), utiliza esse critério para separar o que denomina por conhecimento científico do não-científico. Fazendo um paralelo com o Big Data, o critério de falseabilidade do filósofo não apresenta utilidade, pois a construção de conhecimento a partir do Big Data não se baseia no teste para refutação de teorias. Além disso, devido a componente de velocidade, presente no contexto de Big Data, as teorias por si só podem ser refutadas à medida que novos enunciados singulares sejam incorporados e, consequentemente, novos enunciados universais surjam dentro do processo de indução clássico presente na análise do Big Data (POPPER, 1972).

Outro aspecto importante apresentado por Popper (1972), ainda na discussão sobre a falseabilidade está ligado ao "poder" das teorias com relação em estabelecer a verdade ou falsidade. Segundo Popper, uma decisão positiva a partir de um teste somente valida temporariamente uma teoria, pois decisões subsequentes podem destruí-la. Se uma teoria resiste aos testes durante o curso do progresso científico, pode-se dizer que ela corrobora com a experiência passada (POPPER, 1972).

A luz do Big Data, poder-se-ia concordar com Popper (1972), quanto a questão da validade temporária da teoria, pois os $3 \mathrm{Vs}$ presentes no Big Data, sinalizam que as mudanças quanto ao progresso científico devem ser mais constantes e em maior velocidade. No entanto, pode-se discordar Do filósofo quanto ao fato de que não há como garantir a verdade ou falsidade de uma teoria, pois a mesma sempre estará sujeita a ser falseada.

Se resgatarmos a ideia de que no contexto do Big Data temos todos enunciados singulares presentes quando da concepção de uma nova teoria, pode-se garantir, portanto, a verdade ou falsidade de uma teoria através do método indutivo, assim como validá-la através de um método dedutivo. Embora essa validade seja considerada temporária, corroborando com ideia de Popper (1972), tal validade está diretamente ligada aos 3Vs do Big Data e, consequentemente, a incorporação de novos enunciados singulares ao conjunto de dados levando a crer que a teoria pode ser refutada a partir desse novo conjunto. Porém, ao contrário do que defende Popper (1972), até que a teoria vigente construída através do método indutivo seja refutada pelo novo conjunto composto de todos enunciados singulares, pode-se afirmar que a partir da análise do Big Data a teoria vigente é, portanto, verdadeira.

Popper (1972), permanece na crítica ao indutivismo ao citar os filósofos que defendem que a inferência indutiva pode alcançar um certo grau de confiança ou de probabilidade. Entre os defensores dessa doutrina, a construção do conhecimento científico deve estar amparada 
numa estrutura lógica dos enunciados de forma a garantir a sua consistência. A lógica indutiva está baseada numa teoria de probabilidade lógica que dá sustentação e providência as regras para o pensamento indutivo (LISTON, 2011).

Popper (1972), apresenta uma crítica a esse modelo afirmando que a ideia de probabilidade ou grau de confiança não contribui em nada para a resolução do problema de indução. Partindo do princípio que uma inferência indutiva estará associada a uma certa probabilidade, que não assegurará verdade ou falsidade a essa inferência, tem-se, portanto, uma inferência do tipo "provável", que conduz a uma regressão infinita ou a uma doutrina do apriorismo.

Resgatando a argumentação feita anteriormente e considerando mais uma vez que sob a ótica do Big Data o conjunto dos enunciados singulares representa estatisticamente o conceito de população, uma inferência indutiva a partir desses enunciados poderia ser avaliada como verdadeira ou falsa, atribuindo-se a probabilidade 1 e 0 , respectivamente. Diante dessa constatação, no contexto do Big Data, o grau de confiança e probabilidade, criticadas por Popper, assume os valores que justificam a verdade ou falsidade da inferência indutiva. Dessa forma, o conceito da inferência provável inexiste. Portanto, pode-se considerar também adequada a análise para construção do conhecimento a partir do Big Data sob o ponto de vista defendido por essa doutrina.

\section{CONSIDERAÇÕES FINAIS}

A busca pelo conhecimento e entendimento do universo por parte do ser humano faz parte de uma querela que permanecerá sempre viva. A lógica por trás do pensamento indutivo, que sempre gerou muitos debates e permanece bastante ativa, ganha a partir de agora, um novo componente com surgimento do conceito de Big Data, em especial devido ao retorno das discussões acerca do uso método indutivo na construção do conhecimento científico e, consequentemente, ao progresso da ciência.

O Big Data incorporou uma nova perspectiva à e-Science, a partir da inclusão de características específicas presentes nos seus 3Vs (volume, velocidade, variedade). Diante disso, serve como direcionador de uma nova discussão acerca o problema da indução. O ensaio fez uma análise sob a ótica do Big Data, a partir dos argumentos apresentados pelo filósofo Popper (1972) acerca do problema de indução.

A partir da análise dos argumentos apresentados por Popper (1972) sob a ótica do Big Data, pode-se concluir que a partir dessa reflexão, uma solução alternativa para o problema da 
indução é apresentada a partir do uso do Big Data na pesquisa científica. Na visão do Big Data, o método indutivo não apresenta inconsistências que gerem dúvidas quanto as inferências feitas a partir das experiências, contrariando o que defende Popper (1972) e outros filósofos, que os enunciados universais não podem ser inferidos a partir de enunciados singulares. 


\section{REFERENCIAS}

ALBAGLI, S; APPEL, A. L.; MACIEL, M. L. E-Science e ciência aberta: questões em debate. In: ENCONTRO NACIONAL DE PESQUISA EM CIÊNCIA DA INFORMAÇÃO ENANCIB, 14., 2013, Florianópolis, Anais eletrônicos... Florianópolis: UFSC, 2103. Disponível em:

<http://enancib2013.ufsc.br/index.php/enancib2013/XIVenancib/paper/viewFile/168/362>.

CHEN, J. et al. Big data challenge: a data management perspective. Frontiers of Computer Science, v. 7, n. 2, p. 157-164, 2013.

EDWARDS, P. Russell's doubts about induction. Mind, v. 58, 230, n. p. 141-163, 1949.

GRÁCIO, M. C. C. Sobre a indução. e Prints Cle. 2003. Disponível em:

$<\mathrm{ftp}$ ://ftp.cle.unicamp.br/pub/arquivos/educacional/ArtGT4.pdf>. Acesso em: 20 jun. 2018.

HUME, D. An enquiry concerning humam understanding: a critical edition. New York: Oxford University Press Inc, 2006.

Moeda, 2002.

Investigação sobre o entendimento humano. Lisboa: Imprensa Nacional, Casa da

JUNTA, C. M. John Stuart Mill e a filosofia das ciências sociais. 2011. 88f. Dissertação (Mestrado em Filosofia) - Universidade Federal de São Carlos, São Carlos, 2011.

KAISLER, S. et al. Big data: Issues and Challenges Moving Forward. In: HAWAII INTERNATIONAL CONFERENCE ON SYSTEM SCIENCES, 46., 2013, Wailea, Maui, Proceeding... Wailea, Maui: HI, 2013.

KATAL, A.; WAZID, M.; GOUDAR, R. H. Big Data: Issues, Challenges, Tools and Good Practices. In: INTERNATIONAL CONFERENCE ON CONTEMPORARY COMPUTING (IC3), 6., 2013, Noida, Proceedings, Noida: JIIT, 2013.

KUHN, T. S. The Structure of Scientific Revolutions. University of Chicago Press, 1962.

LINS FILHO, M. L.; SOUZA NETO, M. V. Desafios na era de Big Data: uma revisão. SIMPÓSIO DE ENGENHARIA DE PRODUÇÃO, 21., 2014, Bauru, Anais.. Bauru: UNESP, 2014.

MADDEN, S. From Databases to Big Data. IEEE Internet Computer Society. v. 16, n. 3, p. 4-6. May-Jun, 2012.

NATURE. Big Data: science in petabytes era. Nature Journal, v. 455, n. 7209, 2008.

OLIVA, A. (org.) Epistemologia: a cientificidade em questão. Campinas: Papirus, 1990.

POLYA, G. A arte de resolver problemas. Rio de Janeiro: Interciência, 1978.

POPPER, K. R. A lógica da pesquisa científica. São Paulo: Cultrix, 1972.

. Conjecturas e refutações. 3. ed. Brasília: UnB, 1981. 
REICHENBACH, H. Probability and induction. In: REICHENBACH, H. Experience and prediction. Chicago: University of Chicago Press, 1938. cap. 5, p. 297-403.

SAGIROGLU, S.; SINIANC, D. Big Data: a review. In: INTERNATIONAL CONFERENCE ON COLLABORATION TECHNOLOGIES AND SYSTEMS (CTS), 2013, San Diego CA, Proceeding... San Diego: IEEE, 2013.

SILVEIRA, F. L.; OSTERMANN, F. A insustentabilidade da proposta indutivista de "descobrir a lei a partir de resultados experimentais". Caderno Catarinense de Ensino de Física, Florianópolis, v. 19, n. Especial, p. 7-27, 2002.

SINGH, S.; SINGH, N. Big Data Analytics. In: INTERNATIONAL CONFERENCE ON COMMUNICATION, INFORMATION \& COMPUTING TECHNOLOGY (ICCICT), 2012, Mumbai, Proceeding... Mumbai: IEEE, 2012.

VIANA, G. V. R.; PEREIRA, E. S. O método indutivo. Revista Científica Faculdades Lourenço Filho, v. 5, n. 1, 2007. Disponível em:

$<$ https://www3.ufpe.br/moinhojuridico/images/ppgd/3.0b\%20metodo_indutivo_gerardo_vian a.pdf>. Acesso em: 12 maio 2018. 